\title{
SPACEBORNE P-BAND MIMO SAR FOR PLANETARY APPLICATIONS
}

\author{
Rafael Rincon ${ }^{1}$, Lynn Carter ${ }^{2}$, Daniel Lu ${ }^{1}$, Martin Perrine ${ }^{1}$, Cornelis Du Toit ${ }^{1}$ \\ ${ }^{1}$ NASA Goddard Flight Center, ${ }^{2}$ University of Arizona
}

\begin{abstract}
SESAR (Space Exploration Synthetic Aperture Radar) is a next generation P-band beamforming radar instrument concept that will enable a new class of observations suitable to meet Decadal Survey science goals for planetary exploration. The radar operates at full polarimetry and fine (meter scale) resolution, and achieves beam agility through programmable transmit waveforms and digital beamforming on receive. The radar is based on a low power, lightweight design approach conceived to meet the stringent planetary instrument requirements. This instrument concept has the potential to provide unprecedented surface and nearsubsurface measurements of planetary bodies including the Moon, Mars, and asteroids.
\end{abstract}

Index Terms — SAR, beamforming, planetary.

\section{INTRODUCTION}

The Space Exploration Synthetic Aperture Radar (SESAR) is an advanced P-band (70 cm wavelength) radar instrument with capabilities beyond current planetary instruments [1] capable of provide unprecedented planetary surface and near-subsurface measurements. The instrument is well suited to meet a number of Decadal Survey Science Goals that require fine resolution views of subsurface stratigraphy, and the ability to expose bedrock and search for buried features that hold clues about the geologic history. For example, locating habitable regions, finding water, and determining the hydrology and cryosphere evolution is a primary goal of the Mars exploration. SESAR's long wavelength signals would penetrate through meters of material, image buried surfaces [2] at fine spatial resolution and full polarimetry, and provide information to identify signatures of buried ice and water [3].

On the Moon, SESAR would be able to image through meters of surface-covered regolith and provide information to characterize the near-surface stratigraphy and geology, crucial in the understanding of lunar processes and in the identification of landing sites for future lunar missions. SESAR's fine resolution mapping and polarimetry would also provide details about the volcanic processes that built the lunar mare and to locate and track lava tubes [4], important for both science and future exploration purposes.

SESAR will achieve its measurement goals through an operation characterized by full polarimetry, high resolution $(<6 \mathrm{~m})$, and programmable beams. The instrument design is based on a modular, low power, lightweight architecture that allows the customization of the instrument configuration for specific planetary body, such as Mars or the Moon. Advanced features, such as multiple transmit and receive (T/R) channels, programmable waveform generation, and digital beamforming provides SESAR with enhanced capabilities to help answer key questions in planetary science.

\section{INSTRUMENT ARICHITECTURE}

SESAR employs a multiple-input multiple-output (MIMO) and modular approach that distributes the radar systems into instrument panels composed of "smart" active subarrays, as illustrated in Figs 1 and 2. The radar architecture is fully programmable and capable of multi-mode radar operation including polarimetric SAR imaging, nadir SAR altimetry, and scatterometry. Some of its advanced programmable features include single, dual, or full polarimetry; multi-look angle data collection; simultaneous left and right of the track imaging; selectable resolution and swath width; digital beam steering (no moving parts); and beam pattern control; among others. This radar design is based on the successful P-band EcoSAR and L-band DBSAR airborne radars that were developed at the NASA Goddard Space Flight Center [5], [6]. Table 1 lists some of SESAR's main characteristics.

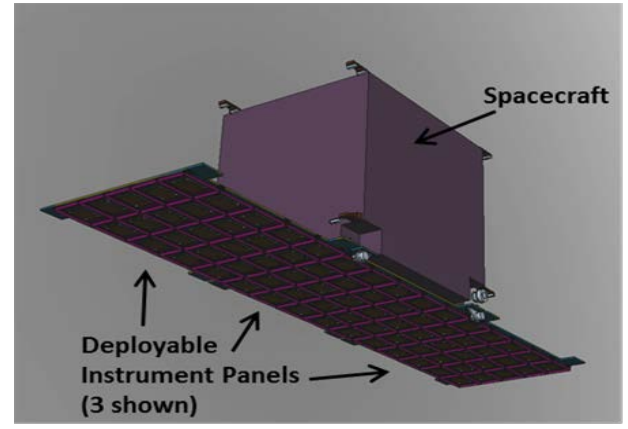

Figure 1 SESAR employs a modular approach that permits customizing the architectures for a given planetary mission. 


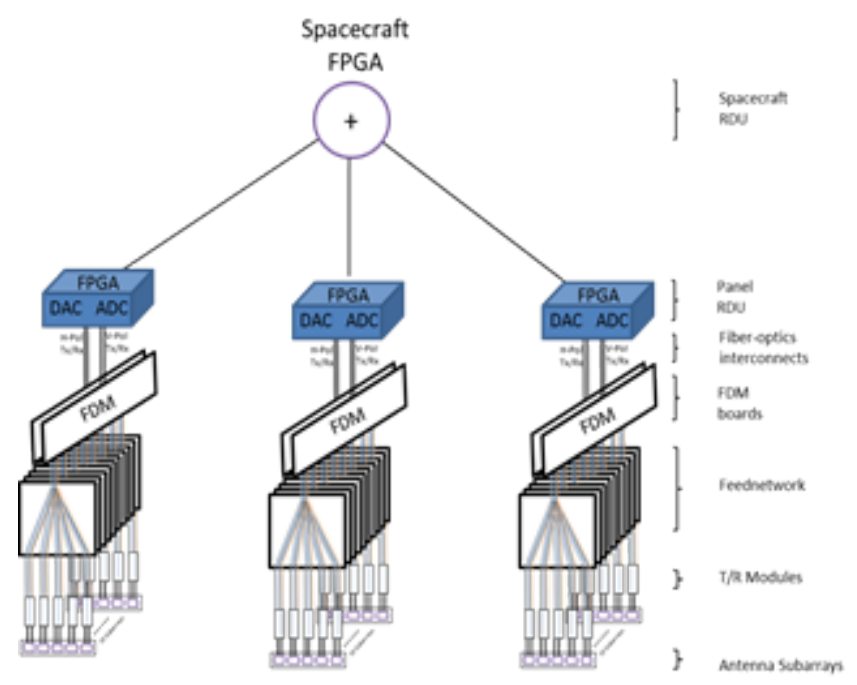

Figure 2 SESAR architecture distributes digital and RF electronics over arrays of antenna elements to provides system flexibility, reduce power, and weight.

SESAR will use a distributed digital and RF electronics architecture that implements advanced waveform modulation techniques to provide the full beam steering agility while significantly reducing the system power consumption. Under the technology-development program awarded by NASA's Planetary Instrument Concepts for the Advancement of Solar System Observations program (PICASSO), the SESAR team is developing innovative techniques to reduce the mass and power consumption of the radar. Through other R\&D efforts, the SESAR team is also developing innovative techniques to reduce the mass of the antenna, and increase the efficiency of the RF electronics.

SESAR's highly innovative architecture will allow synthesis of multiple antenna beams, simultaneously or interleaved, enabling the implementation of non-conventional imaging that can overcome fundamental limitations of conventional radar systems [7],[8],[9]. Some of its benefits include an increase in the measurement swath without reducing the received antenna gain, and the suppression of ambiguities or localized interference in the receiver signal by appropriate null-steering of the antenna pattern. The antenna gain, beam pointing angle, and sidelobe structure can be programmed in real-time for specific tasks. Furthermore, multiple beams can be synthesized on both sides of the flight-track, as well as nadir, using a single nadir-looking antenna, thus increasing the coverage area.

\section{SYSTEM CHARACTERISTICS}

SESAR employs a modular concept that permits the configuration of the radar instrument to meet specific mission requirements. This is possible by the modularity of its four main subsystems: A) the Antenna arrays, B) the RF Transceiver Modules (RTMs), C) the Radar Digital Units (RDUs), and D) the Frequency Domain Multiplexing electronics (FDM), as illustrated in Fig. 2.

\section{A. Antenna Arrays:}

SESAR employs arrays of antenna elements distributed over antenna panels, as shown in Figs 2 and 4. The panels stow around the spacecraft for during launch, and once deployed form a large array. The number of panels employed depend on the mission's target and measurement requirements. After deployment, the antenna is oriented towards nadir, as illustrated in Fig. 2, permitting beamforming across the track over a large field of view $\left(>45^{\circ}\right)$. The antenna elements are based on a stacked-patch element design (see Fig 3) that permits horizontal (H) and vertical (V) polarization measurements over a $100 \mathrm{MHz}$ bandwidth, and crosspolarization isolation greater than $30 \mathrm{~dB}$ [10]. The antenna elements are grouped as subarrays aligned in the flight direction, permit cross-track beam steering over a range of \pm 45 degrees.

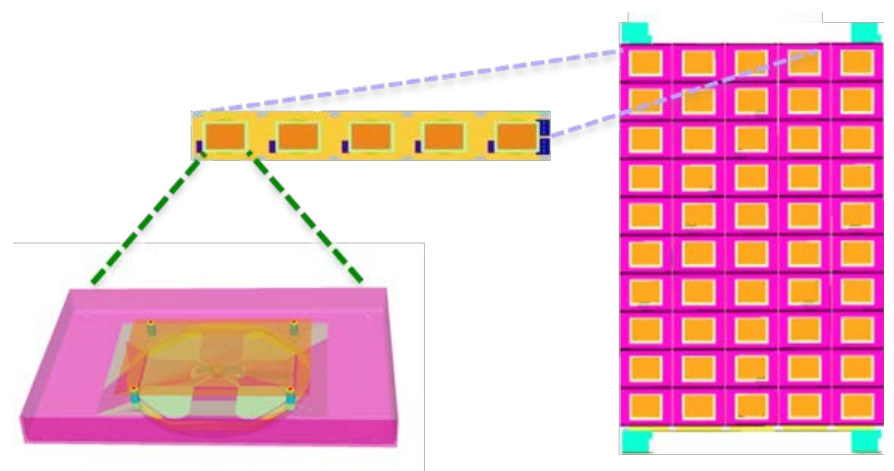

Figure 3. Illustration of SESAR antenna panel architecture. The elements in each antenna form subarrays that enable cross track scanning. The linearly polarized antenna elements ( $\mathrm{H}$ and $\mathrm{V}$ pol) are based on a proven stack-patch design.

\section{B. Radar Transceiver Modules (RTMs):}

SESAR's RTMs build on the heritage of Goddard's Digital Beamforming Synthetic Aperture Radar (DBSAR) and EcoSAR which miniaturized radar transceivers while improving system performance [5] [11]. SESAR's RTMs employ surface mount components on printed circuit board (PCB) which yields a compact and robust design (see Fig. $4)$. 


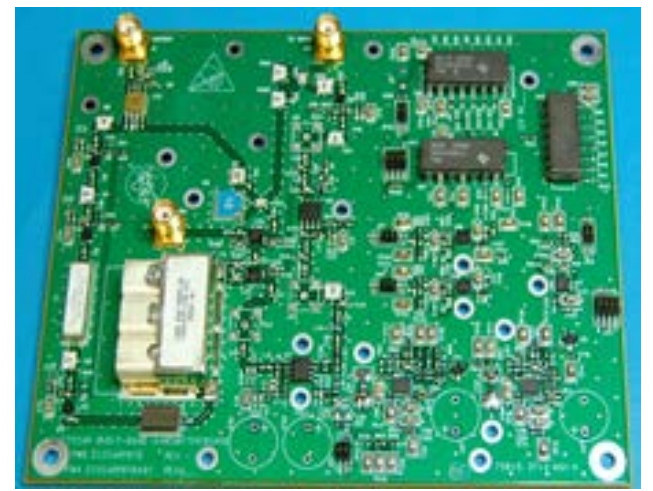

Figure 4 SESAR's compact and highly efficient Radar Transceiver Modules are distributed over the antenna panels driving each of the antenna elements.

The RTMs were designed for high efficiency and light weight. Ongoing efforts with the University of Oklahoma seek to improve the efficiency ( $>50 \%$ ) of the RTMs' solid state amplifiers (SSPA) to further increase system RF power and reduce DC power consumption.

The primary function of the transceivers is to amplify the transmit signal to the necessary power level to feed the antenna subarrays and to amplify the very low power RADAR return signal for processing. In addition, the transceivers provide accurate means to calibrate the transmitter and receiver independently, and to reject out-ofband interference to the receiver.

\section{Radar Digital Units (REUs):}

Each subarray in the radar is controlled by an RDU, as shown in Fig. 5. The RDUs are capable of arbitrary waveform generation, data acquisition, and onboard processing (see fig. 5). These units are designed with Xilinx Kintex 7 FPGAs, low power digital-to-analog converters (D/As) and analog-to-digital converters (A/Ds). They synthesize based band signals with the appropriate phase and amplitude weights for transmit beam steering and side lobe control. Beamforming on receive is performed on-board by coherently acquiring the raw complex data. They are also responsible for system synchronization, radar timing, and data transfers.

\section{Frequency Domain Multiplexing (FDM) Electronics.}

SESAR achieves further reduction in power consumption by employing a novel FDM technique [12]. The FDM approach reduces the number of DACs and ADCs and enables centralized waveform generation and data acquisition with reduced power and mass (see Fig. 5). This approach preserves full array steering capabilities while reducing the number of DACs and ADCs by a factor of 5 or higher, resulting in a significant reduction of SESAR's power consumption.

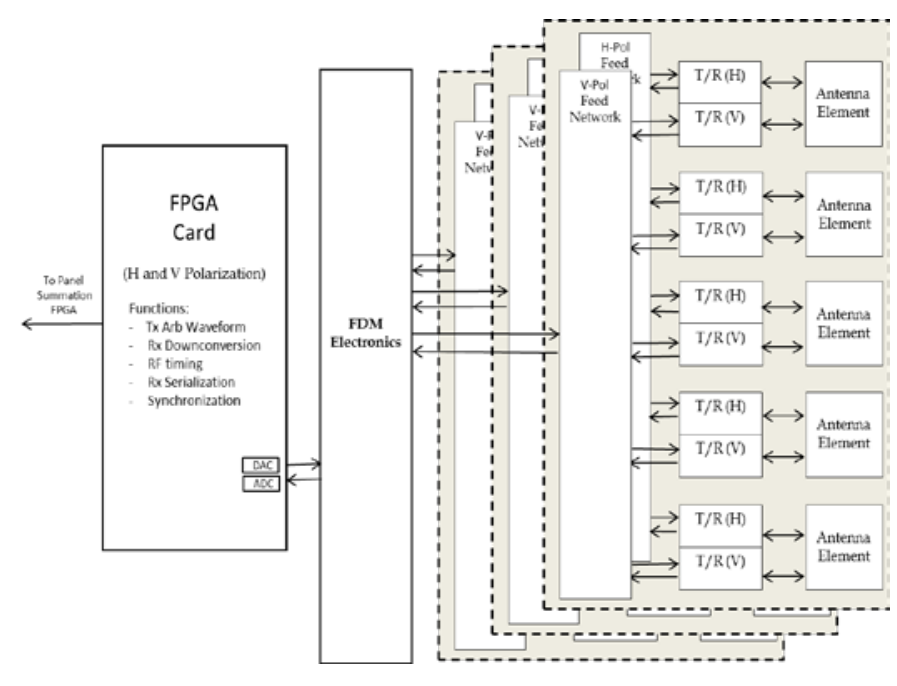

Figure 5. SESAR's RDUs designed for low power consumption, employ Kintex 7 FPGAs, and low power digital-to-analog converters (D/As) and analog-to-digital converters (A/Ds). Significnat power reduction is achieved by employeing a Frequency Domain Mutliplexing (FDM) Technique, which reduces the number of DACs and ADCs in the system.

\section{CONCLUSION}

The SESAR instrument approach would be a first in planetary exploration. SESAR's agile radar operation, modularity, and multimode operation, while using technology that can be optimized to produce the best possible data set for the individual science goals, will help pave the way for the next generation planetary radar systems. SESAR's innovative approach to lower mass and power consumption will make these future missions feasible. Details on each of the subsystems will be given in the final paper.

\section{REFERENCES}

[1] R. Rincon, K. Ranson, T. Fatoyinbo, L. Carter, NASA Patent Application \# GSC-17016-1, Concept Development for Advanced Spaceborne Synthetic Aperture Radar, Sep 28, 2017

[2] P. Paillou, Y. Lasne, E. Heggy, J. Malézieux, G. Ruffié, “A study of P-band synthetic aperture radar applicability and performance for Mars exploration: Imaging subsurface geology and detecting shallow moisture", J. Geophys. Res., Vol. 111, E06S11, doi:10.1029/2005JE002528.

[3] MEPAG NEX-SAG Report, Report from the Next Orbiter Science Analysis Group (NEX-SAG), Chaired by B. Campbell and R. Zurek, posted Dec. 2015 by MEPAG at http://mepag.nasa.gov/reports.cfm. 
[4] B.A. Campbell, B. Hawke, L. M. Carter, R. Ghent, D. B. Campbell, "Rugged lava flows on the Moon revealed by Earth-based radar", Geophys. Res. Lett., 36, 2009 L22201, doi:10.1029/ 2009GL041087.

[5] R.. F. Rincon, T. Fatoyinbo, B. Osmanoglu, S. Lee, C. F. du Toit, M. Perrine, K. J. Ranson, G. Sun, M. Deshpande, J. Beck, D. Lu, and T. Bollian "Digital Beamforming Synthetic Aperture Radar Developments at NASA/Goddard Space Flight Center", IEEE International Symposium on Phased Array Systems and Technology, Waltham, MA, Oct 2016.

[6] R. F. Rincon, T. Fatoyinbo, B. Osmanoglu, S Lee, K. J. Ranson, G. Sun, M. Perrine, C. du Toit, "ECOSAR: P-band digital beamforming polarimetric and single pass interferometric SAR", IEEE Radar Conference (RadarCon), Arlington, VA, May 2015, pp. 0699-0703. doi: 10.1109/RADAR.2015.7131086.

[7] R. F. Rincon, M. Vega, M. Buenfil, A. Geist, L. Hilliard, and P. Racette, "NASA's L-band Digital Beamforming Synthetic Aperture Radar (DBSAR)”, IEEE Trans. Geosci. Rem. Sens., vol.49, no.10, pp.3622-3628, 2011. doi:10.1109/TGRS.2011.2157971.

[8] G. Krieger, N. Gebert, A. Moreira, "Multidimensional Waveform Encoding: A New Digital Beamforming Technique for Synthetic Aperture Radar Remote Sensing”, IEEE Trans. Geosci. Rem. Sens., vol. 46, No. 1, pp. 31 - 46, Jan 2008.

[9] M. Younis, C. Fischer, W. Wiesbeck, "Digital Beamforming in SAR Systems”, IEEE Trans. Geosci. Rem. Sens., vol. 41, No. 71, pp. 1735 -1739 , Jul. 2003.

[10] C. F. du Toit, M. Deshpande, and R.F. Rincon,, "Advanced Antenna Design For Nasa's EcoSAR Instrument”, 2016 IEEE International Symposium on Phased Array Systems and Technology, Waltham, MA

[11] M. L. Perrine; R. Rincon; T. Fatoyinbo; R Zimmerman; N. Spartana; F. Robinson; P. James; S. Seufert; M. Triesky; K. Beltran; and P. Fon, "Development of the RF electronics unit for NASA's ecological synthetic aperture radar”, IEEE International Symposium on Phased Array Systems \& Technology, Oct 2013.

[12] D. Lu and R. Rincon, NASA New Technology Report (NTR), "Frequency Division Multiplexing Scheme for Phasing Synthetic Aperture Radars and Receivers Case No. GSC-17,960-1, Sep 26, 2017. 